ORIGINAL ARTICLE

\title{
Serological speciation of human schistosome infections by ELISA with a panel of three antigens
}

\author{
P Turner, K Lalloo, J Bligh, M Armstrong, C J M Whitty, M J Doenhoff, P L Chiodini
}

J Clin Pathol 2004;57:1193-1196. doi: 10.1136/icp.2003.014779

See end of article for authors' affiliations

.....................

Correspondence to:

Dr P Turner, Department of

Clinical Microbiology,

University College London

Hospital, 3rd Floor A\&E

Building, Grafton Way,

London WC1E 6DB;

pturner@doctors.org.uk

Accepted for publication 1 June 2004
Aims: To find out whether serology can reliably speciate human schistosomiasis using a simple enzyme linked immunosorbent assay (ELISA) technique.

Methods: Stored sera from 66 patients with microscopically confirmed schistosomiasis were subjected to ELISA using a panel of three antigens, namely: unfractionated Schistosoma mansoni soluble egg antigen (SEA); CEF6, a cationic fraction of SEA; and crude $S$ margrebowiei egg antigen, prepared from an animal schistosome closely related to $S$ haematobium.

Results: The optical densities (ODs) obtained using CEF6 as antigen were significantly higher in sera from $S$ mansoni infected patients than in sera from $S$ haematobium infected patients (median OD, $0.810 \mathrm{v}$ 0.595). Using $S$ margrebowiei egg antigen, the optical densities were significantly higher in $S$ haematobium sera than in $S$ mansoni sera (median OD, $0.794 v 0.544$ ). There was no significant difference in optical densities between $S$ mansoni and $S$ haematobium sera using SEA (median OD, 0.725 $v 0.737)$. The ratio of ODs (CEF6 to $S$ margrebowiei egg antigen) was calculated: a ratio of $>1$ indicated $S$ mansoni infection (sensitivity, $88 \%$ ) and a ratio of $<1$ indicated $S$ haematobium infection (sensitivity, $84 \%)$. The odds ratio for $S$ haematobium having an OD ratio of $<1$ was 36.8 (95\% confidence interval, 7.0 to 194).

Conclusions: The identity of the infecting species of schistosome can be determined using the panel of antigens described. SEA should be used to screen serum samples, and the CEF6 : S margrebowiei egg antigen ELISA optical density ratio can be used where serological speciation is required.
S chistosomiasis remains an important infection for people living in and travelling to tropical countries. Figures suggest that there are currently 200 million people suffering from schistosomiasis. ${ }^{1}$

The laboratory diagnosis of schistosomiasis can be achieved by both microscopic and serological methods. ${ }^{2}$ Schistosome ova may be seen in membrane filtered terminal urine, formol/ether concentrated stools, and biopsy specimens. Microscopy has a specificity of $100 \%$ in expert hands, but variable sensitivity. Ova were found in only $45 \%$ of 1107 patients from Africa diagnosed serologically with schistosomiasis in the UK. ${ }^{3}$ There are a variety of serological methods available for the diagnosis of schistosomiasis. The Hospital for Tropical Diseases in London (HTD) uses an enzyme linked immunosorbent assay (ELISA) with crude Schistosoma mansoni soluble egg antigen (SEA), which has a published specificity of $97 \%$ and sensitivities of $96 \%$ ( $S$ mansoni) and 92\% (S haematobium). ${ }^{4}$ The SEA ELISA is a good serological screening test for schistosomiasis, but gives no indication of the infecting species of schistosome. The Centers for Disease Control in the USA screen sera by falcon assay screening test (FAST)-ELISA using $S$ mansoni adult worm microsomal antigen and subsequently confirm the results with a species specific immunoblot. ${ }^{5}$ They report excellent sensitivity and specificity for both the ELISA and the immunoblot.

A cationic fraction of $S$ mansoni egg homogenate, known as CEF6, contains the antigens $\omega-1$ and $\alpha-1 .{ }^{6}$ In several studies, CEF6 has been found to be potentially useful for the serodiagnosis of $S$ mansoni infections. ${ }^{7-10}$ In a large collaborative study, Mott and Dixon reported that ELISA using CEF6 had a sensitivity of $91.7 \%$ and a specificity of $90.0 \%$ for $S$ mansoni infection. ${ }^{11}$ However, Doenhoff and colleagues noted that sera from patients with $S$ haematobium infection significantly crossreacted with CEF6, and were only marginally less reactive against CEF6 than $S$ mansoni sera. ${ }^{10}$ This somewhat limits the usefulness of CEF6 for the specific serodiagnosis of $S$ mansoni infections because there are geographical areas where both $S$ haematobium and $S$ mansoni are endemic. Crossreactivity of sera from $S$ japonicum infected patients with CEF6 has been demonstrated, but this is of less clinical relevance because $S$ japonicum is not known to coexist with $S$ mansoni. ${ }^{7}$

"The speciation of schistosomiasis is important because screening for species specific complications, such as bladder carcinoma in Schistosoma haematobium infection, is necessary in those chronically exposed"

Schistosoma haematobium antigens are not easily obtained: the life cycle of $S$ haematobium is difficult to maintain in the laboratory and egg yields are low. However, a closely related species of schistosome, $S$ margrebowiei, grows well in laboratory mice. Schistosoma margrebowiei commonly infects African antelopes and other herbivores. ${ }^{12}$ Antigens derived from the ova of $S$ margrebowiei might be expected to crossreact with antibodies present in the sera of patients infected with $S$ haematobium, providing a more specific test for $S$ haematobium infection.

Previous work at HTD (M El-Tahawy et al, 1992, unpublished data) has suggested that it is possible to speciate schistosome infections serologically by ELISA using a combination of CEF6 and $S$ margrebowiei egg antigens. Our present study set out to confirm that the infecting species of schistosome could be reliably identified from blood using ELISA with a panel of three antigens, namely: SEA, CEF6,

Abbreviations: ELISA, enzyme linked immunosorbent assay; FAST, falcon assay screening test; HTD, Hospital for Tropical Diseases London; OD, optical density; SEA, Schistosoma mansoni soluble egg antigen 
and $S$ margrebowiei egg antigen. The speciation of schistosomiasis is important because, although the treatment for $S$ haematobium and $S$ mansoni infections is the same, screening for species specific complications, such as bladder carcinoma in $S$ haematobium infection, is necessary in those chronically exposed. Obviously, this is only applicable in a minority of those patients presenting with schistosomiasis in the UK. Species specific serological tests will also be useful in epidemiological studies of schistosomiasis.

\section{METHODS}

Sera from 66 patients, stored after routine diagnostic testing for schistosomal antibodies, were used in our study. The patients were diagnosed with schistosomiasis at HTD between 1989 and 2002. In all patients, the diagnosis had been confirmed by the identification of ova on microscopy of urine, stool, or biopsy material. Appropriate sera were identified by searching the hospital pathology results computer, the parasitology laboratory positive findings book, and a departmental schistosomiasis patient database. All HTD patients with a confirmed microscopic diagnosis of schistosomiasis and an available pretreatment serum sample were included in our study.

All sera were stored at $-20^{\circ} \mathrm{C}$ before analysis.

\section{Preparation of the test antigens}

The SEA and CEF6 antigens were prepared as described by Dunne et al. ${ }^{8}$ Schistosoma margrebowiei was obtained from Malawi and maintained in the laboratory by passage in Bulinus natalensis snails and laboratory mice. Crude $S$ margrebowiei soluble egg antigen was prepared by the same method used by Dunne and colleagues to prepare $S$ mansoni SEA. ${ }^{8}$

The protein concentrations of the CEF6 and $S$ margrebowiei egg antigen preparations were determined using the method described by Lowry et al. ${ }^{13}$ SEA of known concentration was used from the HTD clinical parasitology laboratory antigen stock. The antigen preparations were stored at $-20^{\circ} \mathrm{C}$.

\section{Immobilisation of the test antigens on microtitre plates}

Ninety six well, flat bottomed microtitre plates (Immulon ${ }^{\circledR}$ 2HB; Thermo Labsystems, Franklin, Massachusetts, USA) were used for all of the ELISAs. An appropriate amount of each antigen (as determined by chequerboard titration with reference positive and negative sera) was diluted in coating buffer (carbonate/bicarbonate buffer; $\mathrm{pH}$ 9.6) and $150 \mu \mathrm{l}$ aliquots of these dilutions were dispensed into each well of separate microtitre plates using a multichannel pipette. The plates were covered and incubated at $20-25^{\circ} \mathrm{C}$ for 18 hours. The plates were then washed four times with wash solution $(0.85 \% \mathrm{NaCl}$ and $0.05 \%$ Tween 20$)$ using an automated plate washer $\left(E_{X} 50\right.$ Auto Strip Washer; Bio-tek Instruments Inc, Winooski, Vermont, USA). If not for immediate use, the coated plates were air dried, hermetically sealed, and stored at $4^{\circ} \mathrm{C}$.

\section{ELISA procedure}

Each test serum sample was tested by ELISA using each of the three antigens: SEA, CEF6, and $S$ margrebowiei egg antigen.

The test sera were defrosted rapidly to room temperature. All sera were diluted 1/300 in incubation buffer (phosphate buffered saline and $0.05 \%$ Tween 20; $\mathrm{pH}$ 7.6). The following control sera were used on each plate: a reference positive serum (serum from a patient with microscopically confirmed schistosome infection: $S$ haematobium for the SEA and $S$ margrebowiei egg antigen plates and $S$ mansoni for the CEF6 plates), and a tropical negative serum (serum from an individual with a history of travel to Africa but with no evidence of schistosome infection).

Aliquots of $150 \mu \mathrm{l}$ of each diluted serum sample were dispensed into a separate well on the microtitre plate. The plates were covered and incubated at $26-30^{\circ} \mathrm{C}$ for two hours. After incubation, the plates were washed four times in wash solution. A 1/4000 dilution of peroxidase labelled rabbit antihuman IgG (Dako A/S, Glostrup, Denmark) was prepared in incubation buffer and $150 \mu \mathrm{l}$ aliquots of this dilute conjugate were added to each well. The plates were covered and incubated for a further two hours at $26-30^{\circ} \mathrm{C}$.

The plates were washed a further four times with wash solution. A peroxidase substrate was freshly prepared at this stage $\left(0.1 \mathrm{mg} / \mathrm{ml} \mathrm{OPD}+0.003 \% \mathrm{H}_{2} \mathrm{O}_{2}\right.$ in phosphate/citrate buffer; pH 5.0). Aliquots of $150 \mu \mathrm{l}$ of substrate were added to each well. The optical density (OD) of the positive control sample was monitored at $490 \mathrm{~nm}$ using a Ceres 900i ELISA reader (Bio-tek Instruments Inc) until the target value was reached. The reactions were stopped by adding $30 \mu \mathrm{l}$ of $2.5 \mathrm{M}$ $\mathrm{H}_{2} \mathrm{SO}_{4}$ to each well and the end point optical densities measured: a final reading was taken when the reference positive OD reached its target value (calculated during the chequerboard experiment: 0.800 for SEA, 1.150 for CEF6, and 1.000 for $S$ margrebowiei egg antigen). All sera were tested in duplicate and the arithmetic mean of the two OD results was calculated.

\section{Statistical analysis}

The data were analysed using STATA ${ }^{\mathrm{TM}}$ version 7 software (STATACorp, College Station, Texas, USA). The two sample Wilcoxon rank sum test was used to compare OD results for each antigen. The OD ratios were compared using the $\chi^{2}$ test and the odds ratios were calculated by logistic regression.

\section{RESULTS}

Table 1 shows the results of the ELISAs using the three test antigens. There was no significant difference in ODs between sera from $S$ haematobium infected patients and sera from those infected by $S$ mansoni using SEA as the ELISA antigen $(p=0.9)$. However, using CEF6 as the antigen, the optical densities were significantly higher in $S$ mansoni sera compared with $S$ haematobium sera $(p=0.03)$. With the $S$ margrebowiei egg antigen, $S$ haematobium sera gave significantly higher optical densities than $S$ mansoni sera $(\mathrm{p}=0.0004)$.

Table 1 Results obtained with the three test antigens SEA, CEF6, and Schistosoma margrebowiei egg antigen in sera from patients with confirmed $S$ haematobium or $S$ mansoni infection

\begin{tabular}{llll}
\hline & S haematobium $(\mathbf{n = 5 0 )}$ & S mansoni $(\mathbf{n}=16)$ & p Value \\
\hline SEA & $0.737(0.635$ to 0.796$)$ & $0.725(0.630$ to 0.799$)$ & 0.9 \\
CEF6 & $0.595(0.478$ to 0.698$)$ & $0.810(0.540$ to 1.100$)$ & 0.03 \\
S margrebowiei egg antigen & $0.794(0.675$ to 0.936$)$ & $0.544(0.389$ to 0.649$)$ & 0.0004 \\
OD & $0.785(0.624$ to 0.910$)$ & $1.307(1.123$ to 1.897$)$ & $<0.00001$ \\
\hline & & \\
\hline
\end{tabular}


Table 2 Optical density ratio $\left(O D_{\mathrm{CEF} 6}: \mathrm{OD}_{S}\right.$ margrebowiei egg antigen) of test sera compared with parasitological diagnosis

\begin{tabular}{lrll}
\hline & \multicolumn{2}{l}{ Microscopic diagnosis } \\
\cline { 3 - 4 } & & S haematobium & S mansoni \\
\hline OD ratio & $>1$ & 8 & 14 \\
& $<1$ & 42 & 2 \\
\hline
\end{tabular}

A ratio of the ODs for each serum sample was calculated $\left(\mathrm{OD}_{\mathrm{CEF} 6}: \mathrm{OD}_{S}\right.$ margrebowiei egg antigen $)$. The median ratio was $<1$ for $S$ haematobium sera and $>1$ for $S$ mansoni sera $(\mathrm{p}<0.00001)$ (table 1$)$. The odds ratio for a $S$ haematobium serum having an OD ratio of $<1$ was 36.8 (95\% confidence interval, 7.0 to 194; $\mathrm{p}<0.001$ ). The odds ratio for $S$ mansoni serum having an OD of $<1$ was 0.27 (95\% confidence interval, 0.01 to $0.14 ; \mathrm{p}<0.001)$. Table 2 shows the distribution of sera according to microscopy result and OD ratio. For $S$ haematobium infection, when the OD ratio was $<1$, the test had a sensitivity of $84 \%$; for $S$ mansoni infection, when the OD ratio was $>1$, the test had a sensitivity of $88 \%$. We calculated that, in this patient group, the OD ratio had a positive predictive value of $95 \%$ for $S$ haematobium and $64 \%$ for $S$ mansoni. Although the presence of either $S$ haematobium or $S$ mansoni ova in clinical samples proves that they are present, it is impossible to rule out mixed infection where one species is not visible microscopically (because the sensitivity of microscopy is poor). Therefore, we think that specificities and negative predictive values would not be reliable and have not quoted them.

\section{DISCUSSION}

Schistosomiasis is a major health issue in the tropics and subtropics. Increasing numbers of cases are being reported in short term visitors to endemic countries. It is important to have accurate and cheap methods of diagnosis.

Microscopic methods of diagnosing schistosomiasis are cheap, but are time consuming and have poor sensitivity, although they have excellent specificity. ${ }^{3}$ Therefore, sensitive serological tests have the potential to increase diagnostic yield, especially in those with light infection who excrete few eggs. Unfortunately, current ELISA techniques using $S$ mansoni soluble egg antigen cannot differentiate between $S$ haematobium and $S$ infections. Serological speciation would be useful in patients who have been diagnosed with schistosomiasis on the basis of clinical features and SEA ELISA, but in whom ova cannot be found in clinical material. The Centre for Disease Control in Atlanta provides serological speciation of schistosomiasis using a combination of FASTELISA and immunoblotting; this technique is both sensitive and specific but the immunoblotting is difficult and expensive to perform. ${ }^{5}$ We feel that this would not be a practical technique in a busy laboratory such as the clinical parasitology laboratory at HTD, where approximately 5000 requests for schistosome ELISA are processed each year.

Our experiments have shown that CEF6 is significantly more specific for $S$ mansoni infections, a result that agrees with previously reported findings. ${ }^{7-10}$ However, there is still some overlap in ODs between $S$ mansoni and $S$ haematobium sera using CEF6 as the ELISA antigen. We have found that if $S$ margrebowiei egg antigen is used, significantly higher ODs are obtained with $S$ haematobium sera. Using both CEF6 and $S$ magrebowiei egg antigens in parallel allows a ratio of ODs to be calculated. We have shown that a ratio of $\mathrm{OD}_{\mathrm{CEF} 6}$ : $\mathrm{OD}_{S}$ margrebowiei egg antigen of $<1$ is highly likely to
Take home messages

- We tested an enzyme linked immunosorbent assay (ELISA) on sera of patients with confirmed schistosomiasis using a panel of three antigens: unfractionated Schistosoma mansoni soluble egg antigen (SEA); CEF6, a cationic fraction of SEA; and crude $S$ margrebowiei egg antigen, prepared from an animal schistosome closely related to $S$ haematobium.

- Using this panel of antigens, we were able to identify the infecting species of schistosome

- SEA should be used to screen serum samples, and the CEF6 : $S$ margrebowiei egg antigen ELISA optical density ratio should be used where serological speciation is required

- This will be useful in microscopically negative patients thought to be chronically infected and thus more likely to be at risk of complications

represent $S$ haematobium infection, when compared with the gold standard of identification of ova in clinical material.

"We have found that if Schistosoma margrebowiei egg antigen is used, significantly higher optical densities are obtained with $S$ haematobium sera"

Relatively small numbers of sera were tested in our study, with most (around three quarters) being from patients with $S$ haematobium infection. The positive predictive value of the OD ratio will be altered by any change in the relative proportions of $S$ haematobium and $S$ mansoni infection in the test group. However, it is reassuring to note that the proportion of patients diagnosed with microscopically confirmed S haematobium infection was similar (68\%) in the large HTD case series reported by Whitty et al. ${ }^{3}$

We are now able to provide reliable serological schistosome speciation in those instances in which it might be useful-for example, in microscopically negative patients thought to be chronically infected and thus more likely to be at risk of complications. We envisage that SEA ELISA will be used as a screening test, followed up with CEF6 and $S$ magrebowiei egg antigen ELISA if microscopy of stool and urine is negative.

\section{ACKNOWLEDGEMENTS}

We are grateful to Dr V Southgate, Natural History Museum, London for providing a life cycle of $S$ margrebowiei and to the Wellcome Trust for financial support for the provision of antigenic material.

\section{Authors' affiliations}

P Turner, K Lalloo, J Bligh, M Armstrong, C J M Whitty, P L Chiodini, The Hospital for Tropical Diseases, Capper Street, London WCIE 6AU, UK

M J Doenhoff, School of Biological Sciences, University of Wales, Deiniol Road, Bangor, Gwynedd LL57 2UW, Wales, UK

\section{REFERENCES}

1 Savioli L, Stansfield S, Bundy DAP, et al. Schistosomiasis and soil-transmitted helminth infections: forging control efforts. Trans R Soc Trop Med Hyg 2002:96:577-9.

2 Hamilton A, Klinkert M, Doenhoff MJ. Diagnosis of schistosomiasis: antibody detection, with notes on parasitological and antigen detection methods. Parasitology 1998;117:S41-57.

3 Whitty CJM, Mabey DC, Armstrong M, et al. Presentation and outcome of 1107 cases of schistosomiasis from Africa diagnosed in a non-endemic country. Trans R Soc Trop Med Hyg 2000;94:531-4.

4 Toswill JHC, Ridley DS. An evaluation of the ELISA for schistosomiasis in a hospital population. Trans R Soc Trop Med Hyg 1986;80:435-8. 
5 Tsang VCW, Wilkins PP. Immunodiagnosis of schistosomiasis. Screen with FAST-ELISA and confirm with immunoblot. Clin Lab Med 1991;11:1029-39.

6 Dunne DW, Lucas S, Bickle Q, et al. Identification and partial purification of an antigen $(\omega-1)$ from Schistosoma mansoni eggs which is putatively hepatotoxic in T-cell deprived mice. Trans R Soc Trop Med Hyg 1981;75:54-71.

7 McLaren ML, Lillywhite JE, Dunne DW, et al. Serodiagnosis of human Schistosoma mansoni infections: enhanced sensitivity and specificity in ELISA using a fraction containing $\mathrm{S}$. mansoni egg antigens $\omega^{-1}$ and $\alpha-1$. Trans $R$ Soc Trop Med Hyg 1981;75:72-9.

8 Dunne DW, Bain J, Lillywhite J, et al. The stage-, strain-, and species-specificity of a Schistosoma mansoni egg antigen fraction (CEF6) with serodiagnostic potential. Trans R Soc Trop Med Hyg 1984;78:460-70.

9 Dunne DW, Jones FM, Doenhoff, MJ. The purification, characterisation, serological activity and hepatotoxic properties of two cationic proteins $(\alpha-1$ and $\omega-1)$ from Schistosoma mansoni eggs. Parasitology $1991 ; 103: 225-36$

10 Doenhoff MJ, Butterworth AE, Hayes RJ, et al. Seroepidemiology and serodiagnosis of schistosomiasis in Kenya using crude and purified egg antigens of Schistosoma mansoni in ELISA. Trans R Soc Trop Med Hyg 1993;87:42-8.

11 Mott KE, Dixon H. Collaborative study on antigens for immunodiagnosis of schistosomiasis. Bull World Health Organ 1982;60:729-53.

12 Pitchford RJ. Preliminary observations on the distribution, definitive hosts and possible relation with other schistosomes, of Schistosoma margrebowiei, Le Roux, 1933 and Schistosoma leiperi, Le Roux, 1955. J Helminthol 1976;50:111-23.

13 Lowry OH, Rosebrough NJ, Farr AL, et al. Protein measurement with the Folin phenol reagent. J Biol Chem 1951;193:265-75.

\section{$\mathrm{ECHO}$}

\section{Childhood onset SLE found to be associated with oestrogen receptor $\alpha$ gene polymorphism}

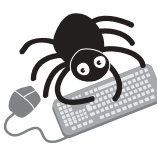

Please visit the Journal of Clinical Pathology website [www. jclinpath.com] for a link to the full text of this article. or the first time, children developing systemic lupus erythematosus (SLE) under the age

- of 16 have been found to have significantly different polymorphisms of the Pvull and

Xbal genes for oestrogen receptor $\alpha(\mathrm{OR} \alpha)$ compared to those developing the disease over the age of 16.

In a case-control study carried out in Korea of 137 female patients with SLE and 268 matched controls, 41 cases developed the disease under the age of 16.

Genomic DNA was extracted from the peripheral blood of all participants, and Pvull and Xbal restriction fragment length polymorphisms of $\mathrm{OR} \alpha$ investigated using polymerase chain reaction primers.

$\mathrm{PP}, \mathrm{Pp}$ and $\mathrm{xx}$ genotypes occurred significantly less frequently in cases developing SLE in childhood than in controls $(\mathrm{p}=0.005,0.05$, and 0.026 respectively). The PP genotype also occurred significantly less frequently in childhood onset than adult onset SLE $(p=0.016)$. Additionally patients with the $\mathrm{Xx}$ genotype had earlier onset of SLE than those with $\mathrm{xx}$ genotype $(p=0.025)$. The frequency of the combined $p p X x$ genotype was significantly greater in childhood onset SLE than in controls $(p=0.0009)$ or adult onset SLE $(p=0.027)$.

PvuII and Xbal polymorphisms of $\mathrm{OR} \alpha$ have already been investigated in other diseases, but this is the first time it has been studied in childhood onset SLE. The authors conclude that this indirectly supports the concept that oestrogen is involved in the pathogenesis of SLE. However the study was limited by the small number of cases of childhood onset SLE. Larger studies in different populations are now needed.

$\Delta$ Lee YJ, et al. Annals of the Rheumatic Diseases 2004;63:1244-1249. 